Recherches en histoire de l'art, histoire des civilisations, archéologie, anthropologie et muséologie

\title{
Exposer la sculpture dans l'Albertinum de Dresde : passé, présent et futur
}

\section{Astrid Nielsen}

Traducteur : Coralie Zermatten

\section{(2) OpenEdition}

\section{Journals}

Édition électronique

URL : http://journals.openedition.org/cel/339

DOI : $10.4000 /$ cel.339

ISSN : 2262-208X

Éditeur

École du Louvre

\section{Référence électronique}

Astrid Nielsen, «Exposer la sculpture dans l'Albertinum de Dresde : passé, présent et futur », Les

Cahiers de l'École du Louvre [En ligne], 8 | 2016, mis en ligne le 01 mai 2016, consulté le 17 septembre 2019. URL : http://journals.openedition.org/cel/339 ; DOI : 10.4000/cel.339

\section{(c) (i) (9)}

Les Cahiers de l'École du Louvre sont mis à disposition selon les termes de la licence Creative Commons Attribution - Pas d'Utilisation Commerciale - Pas de Modification 4.0 International. 


\title{
Cahiers de l'École du Louvre
} recherches en histoire de l'art, histoire des civilisations archéologie, anthropologie et muséologie

\section{Numéro 8. Mai 2016}

\author{
Exposer la sculpture dans l'Albertinum \\ de Dresde : passé, présent et futur \\ Astrid Nielsen
}

Article disponible en ligne à l'adresse :

http://www.ecoledulouvre.fr/cahiers-de-l-ecole-du-louvre/numero8-mai2016/Nielsen.pdf

Pour citer cet article :

Astrid Nielsen, «Exposer la sculpture dans l'Albertinum de Dresde : passé, présent et futur », Cahiers de I'École du Louvre. Recherches en histoire de I'art, histoire des civilisations, archéologie, anthropologie et muséologie [en ligne] n 8, mai 2016, p. 41 à 50 .

\section{@creative \\ ㅇ.. $\odot \circledast$}

(c) École du Louvre

Cet article est mis à disposition selon les termes de la Licence Creative Commons Attribution - Pas d'utilisation commerciale - Pas de modification 3.0 non transposé. 


\section{Cahiers de l'École du Louvre}

recherches en histoire de l'art, histoire des civilisations archéologie, anthropologie et muséologie

\section{Numéro 8. Mai 2016}

\section{Sommaire}

\section{Éditorial}

\section{Articles}

Malcolm Baker, Distinguished Professor of the History of Art, University of California, Riverside Writing about displays of sculpture: historiography and some current questions....

Catherine Chevillot, Directrice du Musée Rodin

Les enjeux d'une rénovation.

Dominique Brard, Architecte

Le Musée Rodin de 2015 : choix muséographiques

p. $20-28$

Geneviève Bresc-Bautier, Conservatrice générale du patrimoine honoraire Exposer la sculpture au Louvre au temps de Rodin, 1860-1914 p. $29-40$

Astrid Nielsen, Conservatrice des collections de sculpture, Albertinum, Dresde Exposer la sculpture dans l'Albertinum de Dresde : passé, présent et futur.....p. 41-50

Colin Lemoine, Historien de l'art, responsable des sculptures au musée Bourdelle

Amélie Simier, Conservatrice en chef du patrimoine, directrice des musées Bourdelle et Zadkine

La sculpture sur le bout des doigts. Retour sur l'élaboration

d'une salle pédagogique et tactile au musée Bourdelle.....

Cecilia Hurley-Griener, Docteur en histoire de l'art (HDR), équipe de recherche, École du Louvre

Putting sculpture on show/Exposer la sculpture : conclusion. p. $58-65$ 


\section{Exposer la sculpture dans l'Albertinum de Dresde : passé, présent et futur}

\section{Astrid Nielsen}

L'Albertinum de Dresde fut rouvert en 2010 après quatre années de fermeture nécessaires aux travaux de réhabilitation et de rénovation pour réparer les dégâts causés par l'inondation de 2002. Dans ce nouveau "Musée des Modernes" ", la Collection des Sculptures et la Galerie des Nouveaux Maîtres exposent des œuvres de l'époque romantique à nos jours dans l'ensemble du bâtiment et dans un nouvel agencement (fig. 1). On trouve des peintures de Caspar David Friedrich (1774-1840), de Paul Gauguin (1848-1903), de Ernst Ludwig Kirchner (1880-1938) ou de Gerhard Richter (né en 1932) placées au premier étage et des sculptures d'Ernst Rietschel (1804-1861), d'Auguste Rodin (18401917) ou de Max Klinger (1857-1920) jusqu’à des travaux de sculpteurs de notre époque $^{2}$. Cette contribution doit tout d'abord livrer un bref aperçu de l'histoire de la Collection des Sculptures de l'Albertinum puis détailler le développement de la conception de l'exposition des oeuvres de la Collection des Sculptures et, pour terminer, les perspectives pour l'avenir du musée seront envisagées. En effet, durant les années à venir, les œuvres de notre époque devraient être plus fortement mises en avant, ce qui devrait renforcer encore l'idée d'un Albertinum comme Musée des Modernes durant le XxI ${ }^{\mathrm{e}}$ siècle.
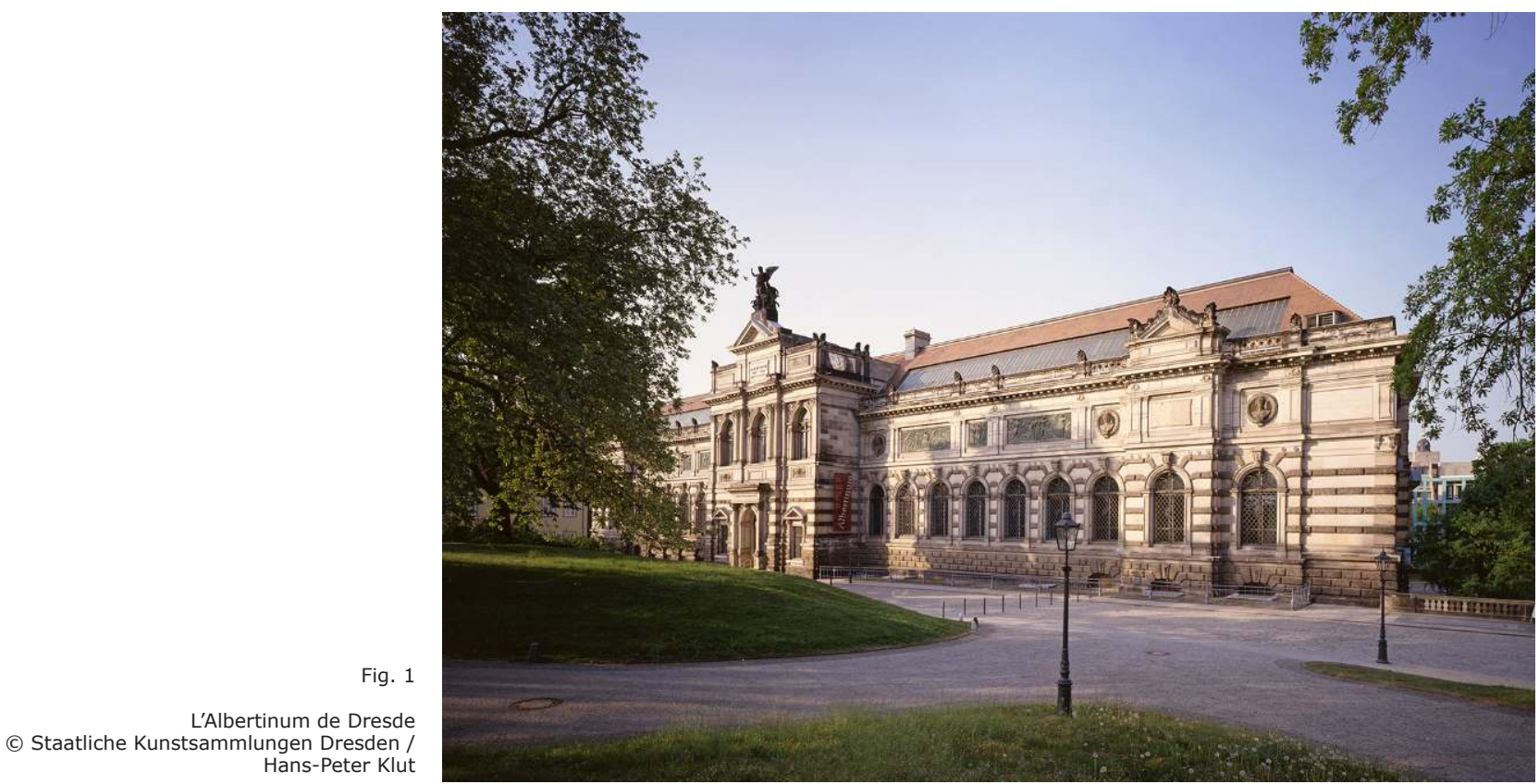

"La Collection des Sculptures comprend plus de cinq millénaires d'œuvres d'art de toutes les époques de la plastique européenne, depuis l'Antiquité en passant par le Moyen Âge et jusqu'à nos jours. Le cœur de la collection se trouve dans l'important fonds d'antiques avec des sculptures comme le Garçon de Dresde ainsi que des vases, des bronzes et des terre-cuites ${ }^{3}$ ». Pour le moment, la collection

1. Gegen den Strom. Die Rettung der Dresdner Kunstschätze vor dem Hochwasser im August 2002, sous la direction de Caroline Möhring et Johannes Schmidt (pour les Staatlichen Kunstsammlungen Dresden), Cologne, König, 2002; Das neue Albertinum : Eine Arche für die Kunst. Depotneubau und Umbau im Albertinum - eine Projektskizze / The New Albertinum : An Ark for Art. New Workshop and Storage Facility Construction - A Project Sketch, Staatliche Kunstsammlungen Dresden / Staatsbetrieb Sächsisches Immobilien- und Baumanagement (SIB), Dresden, Meißen, 2007.

2. Voir Moritz Woelk, «Das neue Albertinum », Das neue Albertinum. Kunst von der Romantik bis zur Gegenwart, sous la direction d'Ulrich Bischoff et Moritz Woelk, Berlin/München, Deutscher Kunstverlag, 2010, pp. 13-20.

3. http://www.skd.museum/de/museen-institutionen/albertinum/skulpturensammlung/index. html (consulté en décembre 2015). 
d'antiques est rassemblée dans les différents dépôts de l'Albertinum ${ }^{4}$, mais elle trouvera un nouveau domicile en 2019.

\section{Brèves remarques sur I'histoire de la collection et du musée}

La Collection des Sculptures de Dresde grandit depuis le $\mathrm{XvI}^{\mathrm{e}}$ siècle, raison pour laquelle il importe de jeter un regard sur le passé pour comprendre la nouvelle conception de ces dernières années. Les origines apparaissent avec le cabinet d'art du prince électeur Auguste (1526-1586) en $1560^{5}$ puis, un agrandissement d'importance équivalent à une nouvelle fondation eut lieu avec Auguste le Fort (1670-1733), prince électeur de Saxe et roi de Pologne ${ }^{6}$. En 1728, certaines œuvres de la succession du prince Augusto Chigi ainsi que des pièces de la collection du Cardinal Alessandro Albani (1692-1779) ont pu être acquises à Rome ${ }^{7}$. Dans le même temps, des sculptures de l'époque furent aussi collectionnées, correspondant ainsi aux pièces baroques allemandes, françaises et italiennes. Entre 1729 et 1747 , cette collection était conservée dans le Palais du Grand Jardin puis dans le Palais japonais. Avec l'achat, durant l'année 1783, de 833 moulages en plâtre de la succession du peintre Anton Raphaël Mengs (17281779), la collection prit l'aspect supplémentaire d'une collection de moulages comptant au milieu du XIX ${ }^{\mathrm{e}}$ siècle environ 4500 pièces $^{8}$. Par ailleurs, la collection des originaux, se complétait au fil du temps avec des œuvres de l'Antiquité grecque et romaine ainsi que des œuvres d'artistes contemporains.

Depuis 1894, la Collection des Sculptures est conservée à l'Albertinum, construit comme armurerie pour la ville de Dresde entre 1559 et 1563. Après la construction d'un nouvel arsenal en 1877, l'armurerie perdit son usage premier. Ainsi, le Parlement saxon décréta en 1884 que la collection d'antiques et de moulages, qui prendrait trois ans plus tard le nom de "Collection des Sculptures ", serait hébergée, avec les archives centrales, dans l'ancienne armurerie. Les travaux de reconstruction du bâtiment se poursuivirent jusqu'en 18899. Il reçut l'apparence qui est toujours la sienne aujourd'hui, de style néo-renaissance, fut nommé en référence au roi Albert (1828-1902) et devint le musée de cette Collection des Sculptures. En 1891, la collection de moulages fut inaugurée au deuxième étage, et trois ans plus tard celle des sculptures originales au premier étage ${ }^{10}$. À partir de ce moment, la collection s'agrandit constamment d'œuvres sculptées contemporaines des périodes traversées. Ainsi, la Collection des Sculptures de Dresde est le premier musée allemand qui acquiert en 1894 avec L'Homme au nez cassé et le plâtre de L'Âge d'airain, des œuvres d'Auguste Rodin

4. Kordelia Knoll, «Die Schaudepots der Skulpturensammlung », Dresdener Kunstblätter 4, 2010 (= Themenheft : Das neue Albertinum), pp. 220-223.

5. Sur les collections électorales et royales depuis leurs fondations jusqu'à aujourd'hui : Zukunft seit 1560. Von der Kunstkammer zu den Staatlichen Kunstsammlungen Dresden, sous la direction de Karin Kolb, Gilbert Lupfer et Martin Roth, 3 vol., Berlin, Deutscher Kunstverlag, 2010 ; Gerald Heres, Dresdener Kunstsammlungen im 18. Jahrhundert, Leipzig, Seemann, 2006, pp. 77-84.

6. Voir entre autres : Virginie Spenlé, « Les acquisitions de Raymond Leplat à Paris », cat. d'exp., Splendeurs de la cour de Saxe: Dresde à Versailles, sous la direction de Béatrix Saule et Dirk Syndram, Musée national et Domaine national de Versailles, 23.1.-23.4.2006, Paris, Réunion des Musées nationaux, 2006, pp. 70-79.

7. Voir entre autres : K. Knoll, « Geschichte der "Churfürstlichen Antiken-Galerie in Dresden" », Skulpturensammlung, Staatliche Kunstsammlungen Dresden. Katalog der antiken Bildwerke II : Idealskulptur der römischen Kaiserzeit 1, sous la direction de K. Knoll, Christiane Vorster, M. Woelk, München, Hirmer, 2011, pp. 1-15 ; Beatrice Cacciotti, « Kunsthändler, Antiquare und Antikensammler im Rom zwischen 1700 und 1733 », cat. d'exp., Verwandelte Götter. Antike Skulpturen des Museo del Prado zu Gast in Dresden, sous la direction de Stefan Schröder, Madrid, Museo del Prado, 4.11.2008-12.4.2009 et Dresden, Japanisches Palais, 20.5.-27.9.2009, Dresden, Staatliche Kunstsammlungen Dresden, 2009, pp. 96-105, 359-363.

8. Die Sammlung der Gipsabgüsse von Anton Raphael Mengs in Dresden. Katalog der Abgüsse, Rekonstruktionen, Nachbildungen und Modelle aus dem römischen Nachlass des Malers in der Skulpturensammlung, Staatliche Kunstsammlungen Dresden, édition préparée par Morit Kiderlen, avec des photos de Hans-Peter Klut, München, Biering \& Brinkmann, 2006.

9. K. Knoll, «Der Umbau des Zeughauses zum Skulpturenmuseum », cat. d'exp. Das Albertinum vor 100 Jahren - die Skulpturensammlung Georg Treus : zur Erinnerung an die Eröffnung der Sammlung der Originalbildwerke am 22. Dezember 1894, sous la direction de K. Knoll, Staatliche Kunstsammlungen Dresden, 18.12.1994-12.3.1995, Dresden, Staatliche Kunstsammlungen Dresden, 1994, pp. 46-48.

10. Fondamental : cat. d'exp., Das Albertinum vor 100 Jahren - die Skulpturensammlung Georg Treus : zur Erinnerung an die Eröffnung der Sammlung der Originalbildwerke am 22. Dezember 1894, sous la direction de K. Knoll, Staatliche Kunstsammlungen Dresden, 18.12.1994-12.3.1995, Dresden, Staatliche Kunstsammlungen Dresden, 1994. 
$(1840-1917)^{11}$. À partir de 1900, sous la direction de l'archéologue Georg Treu (1843-1921), la Collection des Sculptures, qui avait la réputation d'être un musée exemplaire de l'histoire de la plastique - " une grande école de la plastique, comme il n'en existe pas d'autre au monde ${ }^{12} »-$ se développe jusqu'au début de la Première Guerre mondiale en un musée singulier et progressiste, un refuge unique de l'art sculptural historique et actuel, dont « le dialogue entre l'art antique et l'art contemporain [...] est le leitmotiv ${ }^{13}$ ". Jusqu'en 1933, de nombreuses et importantes pièces ont pu être acquises. Durant la période nationale-socialiste, des sculptures du département des modernes qualifiées "d'art dégénéré » ont été réquisitionnées et pour la plupart vendues à l'étranger, comme par exemple l'Agenouillée de Wilhelm Lehmbruck (1881-1919) ${ }^{14}$. Avec le début de la Seconde Guerre mondiale, le musée est fermé et les sculptures évacuées, de sorte que la collection a pu traverser l'épreuve de la guerre avec très peu de pertes. Le bâtiment, en revanche, est durement touché par le bombardement de Dresde en 1945, mais sa reconstruction a lieu assez rapidement ${ }^{15}$. Lorsqu'à la fin des années 1950 , les œuvres réquisitionnées par l'armée rouge et envoyées en Union soviétique reviennent à Dresde, l'Albertinum est transformé en un lieu d'exposition central pour les autres musées. Les joyaux de la Voûte Verte, la porcelaine, les trésors du cabinet numismatique et de la Salle d'Armes y étaient montrés. La Collection de Porcelaine et la Salle d'Armes ont été certes à nouveau déménagées, mais les autres œuvres restèrent en place. La première grande exposition des antiques fut inaugurée en 1969 au rez-de-chaussée du hall Renaissance rénovée ${ }^{16}$. Depuis la création de la Galerie des Nouveaux Maîtres en 1959 et son installation dans l'Albertinum en 1963, la présentation des peintures au deuxième étage était toujours accompagnée de quelques sculptures choisies des $\mathrm{XIX}^{\mathrm{e}}$ et $\mathrm{XX}^{\mathrm{e}}$ siècles ${ }^{17}$. Ce principe fondamental ne changea pratiquement pas après la Réunification en 1990. Les salles d'exposition furent rénovées entre 1992 et 1993 et les œuvres d'art présentées ensemble ${ }^{18}$, même si la sculpture moderne reçut moins d'espace que la peinture. Au début de l'année 2001, un nouveau type de présentation de la Galerie des Nouveaux Maîtres et de la Collection des Sculptures fut envisagée afin de transformer l'Albertinum en un musée moderne, englobant les genres et

11. Cf. : cat. d'exp., Vor 100 Jahren : Rodin in Deutschland, sous la direction de Michael Kuhlemann, Hélène Pinet, Christina Buley-Uribe, Hamburg, Bucerius Kunst Forum, 18.2.-25.5.2006, Dresden, Staatliche Kunstsammlungen Dresden, 10.6.-13.8.2006, München, Hirmer, 2006.

12. Julius Elias, « Kunstausstellungswesen und -unwesen », Die Nation, 37, 15 juin 1901.

13. M. Woelk, « Le département des sculptures à l'Albertinum », Dossier de l'art, 98, 2003, Dresde. Les fabuleuses collections des princes de Saxe, pp. 114-119.

14. Sur ce sujet : Dietrich Schubert, Wilhelm Lehmbruck « Kniende » (1911), Berlin/Dresden, Kulturstiftung der Länder, 1994 (Patrimonia, 93); cat. d'exp., 100 Jahre « Kniende ». Lehmbruck mit Matisse, Brancusi, Debussy, Archipenko, Rodin, Nijinsky in Paris 1911, sous la direction de Raimund Stecker et Marion Bornscheuer, Duisberg, Stiftung Wilhelm Lehmbruck Museum, 24.9.2011-22.1.2012, Köln, DuMont, 2011.

15. Werner Schmidt, «Das Albertinum in neuer Gestalt », Vom Klassizismus zum Jugendstil - Die ständige Ausstellung im Albertinum Dresden, sous la direction de Heiner Protzmann \& Werner Schmidt, Dresden, Staatliche Kunstsammlungen Dresden Gemäldegalerie Neue Meister/ Skulpturensammlung, 1993 , pp. 5-8, ici p. 5 ; sur la reconstruction de I'Albertinum et ses expositions voir les rapports de Martin Raumschüssel (Directeur de la Collection des Sculptures de 1959 à 1992) dans le Jahrbuch der Staatlichen Kunstsammlungen Dresden 1959, 1960, $1961 / 1962,1963 / 1964,1965 / 1966,1968 / 1969$

16. Sur ce sujet: Martin Raumschüssel, « Skulpturensammlung », Jahrbuch der Staatlichen Kunstsammlungen Dresden, 1968/1969, pp. 329-335.

17. Horst Zimmermann, «Zur Geschichte der Gemäldegalerie Neue Meister », Vom Klassizismus zum Jugendstil - Die ständige Ausstellung im Albertinum Dresden, sous la direction de Heiner Protzmann \& Werner Schmidt, Dresden, Staatliche Kunstsammlungen Dresden Gemäldegalerie Neue Meister/ Skulpturensammlung, 1993, pp. 9-11, ici p. 9 ; Uta Neidhardt, «Die Dresdener Gemäldegalerie Alte und Neue Meister seit 1939. Auslagerung, Abtransport und Rückkehr ihrer Werke », cat. d'exp., Zurück in Dresden : eine Ausstellung ehemals vermißter Werke aus Dresdener Museen: Skulpturensammlung, Rüstkammer, KupferstichKabinett, Gemäldegalerie Alte Meister, Gemäldegalerie Neue Meister, Kunstgewerbemuseum, Porzellansammlung, sous la direction d'Harald Marx, Dresden, Residenzschloss, 9.4.-12.7.1998, Eurasburg, Edition Minerva, 1998, pp. 128-131.

18. Vom Klassizismus zum Jugendstil - Die ständige Ausstellung im Albertinum Dresden, sous la direction de Heiner Protzmann \& Werner Schmidt, Dresden, Staatliche Kunstsammlungen Dresden Gemäldegalerie Neue Meister/ Skulpturensammlung, 1993 ; Vom Expressionismus zur Gegenwart - Die ständige Ausstellung im Albertinum Dresden, sous la direction de Gabriel Männel, Dresden, Staatliche Kunstsammlungen Dresden Gemäldegalerie Neue Meister/ Skulpturensammlung, 1993. L'exposition des œuvres se faisait surtout sur des socles en bois. Pour les œuvres du classicisme des colonnes en marbre avait été fabriquées et aujourd'hui, vingt ans après, elles semblent désuètes et d'une esthétique dépassée. 
les époques et permettant de faire se rencontrer l'art ancien et l'art contemporain, de l'Égypte ancienne à nos jours ${ }^{19}$.

\section{L'inondation de 2002, modifications et reconstruction à partir de 2006 : vers un nouvel Albertinum comme Musée des Modernes}

En 2002, les inondations ont brutalement changé la situation ${ }^{20}$ (fig. 2). Une menace directe provenait de l'eau des nappes phréatiques qui pénétrait dans les voûtes des caves. La Collection des Moulages s'y trouvait dans un nouvel
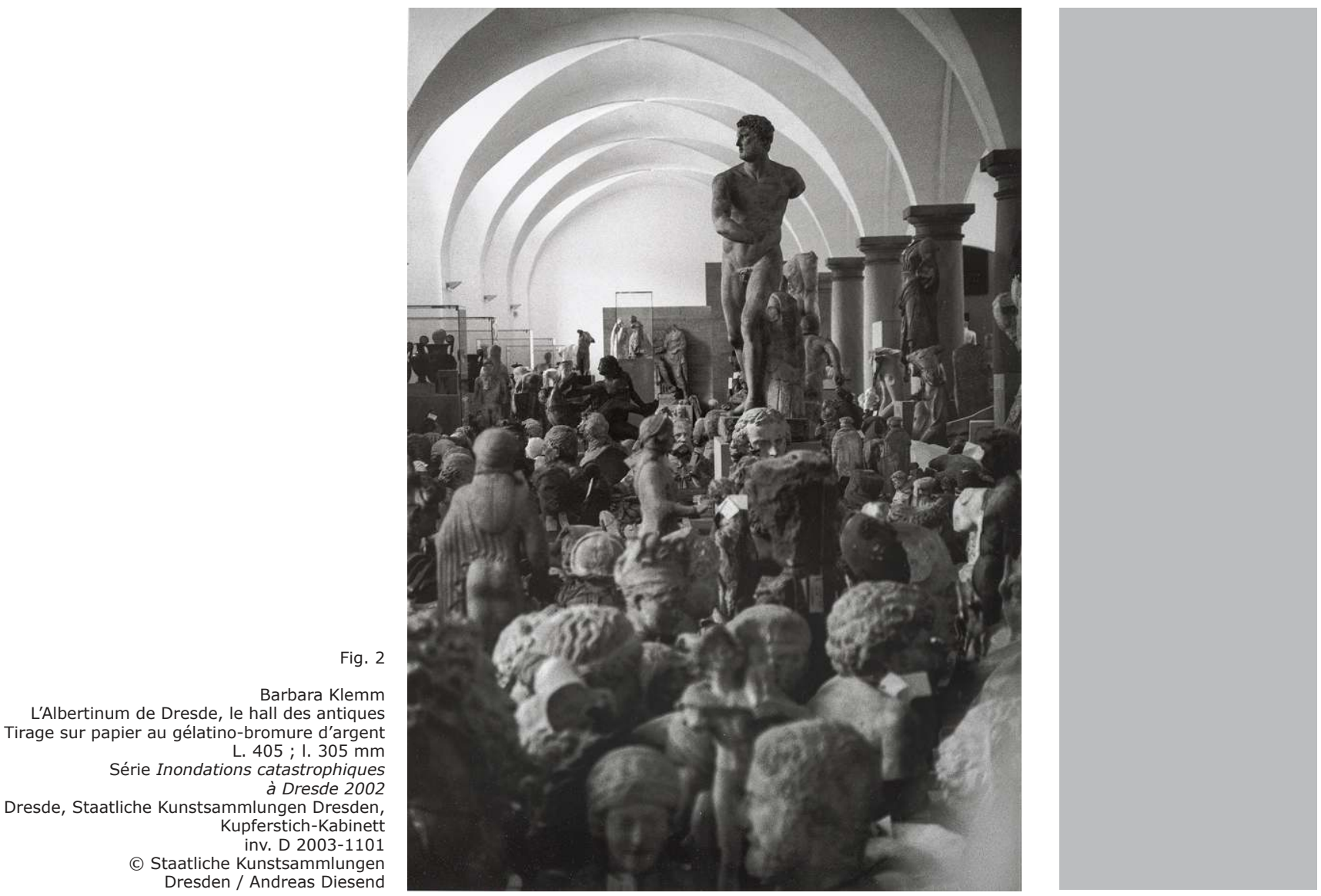

agencement et y était ouverte au public depuis une année seulement depuis la Seconde Guerre mondiale ${ }^{21}$. Elle était aussi menacée que les fonds d'originaux déposés dans les caves. Aussi rapidement que possible, les pièces furent transportées de la cave au rez-de-chaussée de l'Albertinum ${ }^{22}$. L'étendue des dégâts donnait l'occasion de repenser complètement la conception du musée. Il était clair que de nouveaux dépôts dans lesquels les œuvres d'art seraient protégées devenaient nécessaires. Ce qui commença tout d'abord comme une catastrophe se révéla être une chance pour les Collections d'Art de Dresde. En 2004, un concours eut lieu pour la construction d'un nouveau dépôt central avec des ateliers dans la cour intérieure de l'Albertinum ${ }^{23}$. Ce concours fut gagné par l'architecte Volker Staab ${ }^{24}$.

19. M. Woelk, «Skulpturensammlung », Jahrbuch der Staatlichen Kunstsammlungen Dresden, $30,2002 / 2003$, p. 339.

20. Sur ce sujet voir entre autres : cat. d'exp., Nach der Flut. Die Dresdener Skulpturensammlung in Berlin, sous la direction de M. Woelk et K. Knoll, Berlin, Martin-Gropius-Bau, 22.11.200210.2.2003, Leipzig, Seemann, 2002.

21. Die Sammlung der Gipsabgüsse, op. cit. note 8, p. 8.

22. Reproductions entre autres dans : Nach der Flut, op. cit. note 20 ; Gegen den Strom, op. cit. note 1 , en particulier pp. 36-47.

23. http://www.competitionline.com/de/ausschreibungen/1202 (consulté en janvier 2016).

24. Le projet de Staab fut choisi parmi 92 candidatures et fut couronné en 2011 du prix allemand d'architecture. Au total les travaux de construction coutèrent 52 millions d'euros. Voir à ce sujet : https://www.sib.sachsen.de/fileadmin/user upload/pdf-dokumente/broschueren/2012/2011 NL Dresden I Albertinum.pdf, ici p. 47 (consulté en janvier 2016). 
Staab commente son projet comme suit : "Le nouveau bâtiment du dépôt est pensé comme une sorte de toit contenant de l'espace, comme une arche de Noé surélevée, qui dépasserait pour toujours les flots. Sa base est longée de deux rainures laissant filtrer la lumière naturelle tout en la raccordant à la cour intérieure. Il est supporté par une nouvelle cage d'ascenseur et deux piliers situés derrière la façade de la cour conservée [...]. La perspective plafonnante du corps du bâtiment se présente comme une "surface éclairée" puisque la cour intérieure se trouve pleinement éclairée par la lumière qui diffuse les fluctuations de la lueur du jour et les changements entre le soleil et les nuages. Le volume du toit, et ainsi du nouveau corps du bâtiment, se fait alors remarquer dans un second temps. En plus de l'entrée par la terrasse de Brühl, un nouvel accès pour les visiteurs est installé sur la place Georg Treu. Ces deux accès dirigent directement vers la cour intérieure couverte, ce qui souligne encore une fois son rôle de pièce centrale du musée. $[\ldots]^{25}$ "

Des réflexions conceptuelles complètement nouvelles émanaient de ces modifications et de ces reconstructions. L'Albertinum devait devenir un "Musée des Modernes " et le troisième grand complexe de musées dans la structure des Collections d'État de Dresde au côté du Château de la Résidence et du Zwinger ${ }^{26}$. Le profil de l'établissement devait être affiné par l'accent mis sur l'époque romantique dans la peinture et sur la sculpture à partir du XIX siècle jusqu'à aujourd'hui. La présentation des sculptures devait être organisée de manière chronologique, bien que cette idée n'ait pû être développée que pour certaines salles disponibles. La Collection des Sculptures disposait alors du grand hall Renaissance au rez-de-chaussée qui servait auparavant d'espace d'exposition pour la Collection d'Antiques. À ce hall s'ajoutaient deux salles au premier étage : la salle Klinger et la salle dite de la mosaïque. L'installation voulait insister sur les dominantes de la Collection, mais aussi montrer des pièces qui n'avaient que très rarement, ou jamais, été présentées avant.

\section{La sculpture du xVIII ${ }^{\mathrm{e}}$ tardif et du XIX $^{\mathrm{e}}$ siècle}

C'est dans ces deux espaces, la salle de la mosaïque et la salle Klinger, qui se font symétriquement face, que commence la visite de la Collection des Sculptures (fig. 3). Comme ces deux pièces étaient déjà consacrées à la sculpture à la fin du XIX ${ }^{e}$ siècle et que la disposition des cloisons ne permettait d'accrocher que peu de peintures, les sculptures devaient alors y retrouver leur place. Ces deux salles se distinguent des autres pièces d'exposition par leur organisation. Elles étaient autrefois décorées de riches peintures sur les murs et aux plafond ${ }^{27}$, après la destruction de 1945, l'état original ne fut pas reconstitué.

Aujourd'hui, la salle de la mosaïque se présente sous un aspect coloré sobre. Elle est nommée ainsi en raison de la mosaïque de sol provenant de bains romains d'Italie centrale ${ }^{28}$. Cette mosaïque fut posée lorsque les salles furent aménagées en 1894 pour recevoir la collection des originaux antiques ${ }^{29}$. Dans cette salle, la sculpture classique est exposée avec des ouvres de Bertel Thorvaldsen (17681843), Johann Gottfried Schadow (1764-1850) et Christian Daniel Rauch (1777-1857), mais aussi avec des travaux d'Ernst Rietschel (1804-1861), un élève de Rauch et l'un des sculpteurs principaux de Dresde durant le XIX ${ }^{e}$ siècle. Ces œuvres transmettent, de façon exemplaire, tous les idéaux artistiques qui prennent leurs racines et leur orientation dans l'art antique : « élégance et grâce, beauté et

25. http://www.staab-architekten.com/ (Albertinum Dresden - Rénovation et réhabilitation du dépôt central) ; http://www.david-brandt.de/page2/page8/page8.php (consulté en janvier 2016).

26. M. Woelk, op. cit. note 2, pp. 13-20, ici p. 14 .

27. Reproductions dans: Das Albertinum vor 100 Jahren, op. cit. note 10.

28. La mosaïque fut achetée en 1877 après sa découverte en 1843 près de Civitavecchia en Italie. Sur ce sujet : cat. d'exp., Götter und Menschen. Antike Meisterwerke der Skulpturensammlung, sous la direction de Kordelia Knoll, Dresden, Albertinum, 11.2.-30.6.2000, Dresden, Staatliche Kunstsammlungen, 2000, cat. 103, pp. 74 sq. ; Anna Dyroff et Heike Richter, «Das dionysische Bodenmosaik im Albertinum », Restauro, 120, 3, avril-mai 2014, pp. 52-55.

29. K. Knoll, « Die Abnahme der Ergänzungen von den antiken Skulpturen und die Einrichtung und Aufstellung der Antikensammlung », Das Albertinum vor 100 Jahren, op. cit. note 10, pp. 131-134. 
jeunesse, fermeté des contours et la pureté du marbre blanc ${ }^{30} »$. C'est justement ce point commun avec la mosaïque romaine qui révèle cette harmonie. La décision d'exposer aussi des ébauches en plâtre et des modèles originaux d'Ernst Rietschel insiste sur le fait que de nombreuses pièces, qui jusque-là étaient conservées en dépôt, étaient enfin montrées au public. Ernst Rietschel est également connut pour sa création du monument de Johann Wolfgang von Goethe (1749-1832) et Friedrich Schiller (1759-1805) à Weimar ${ }^{31}$.

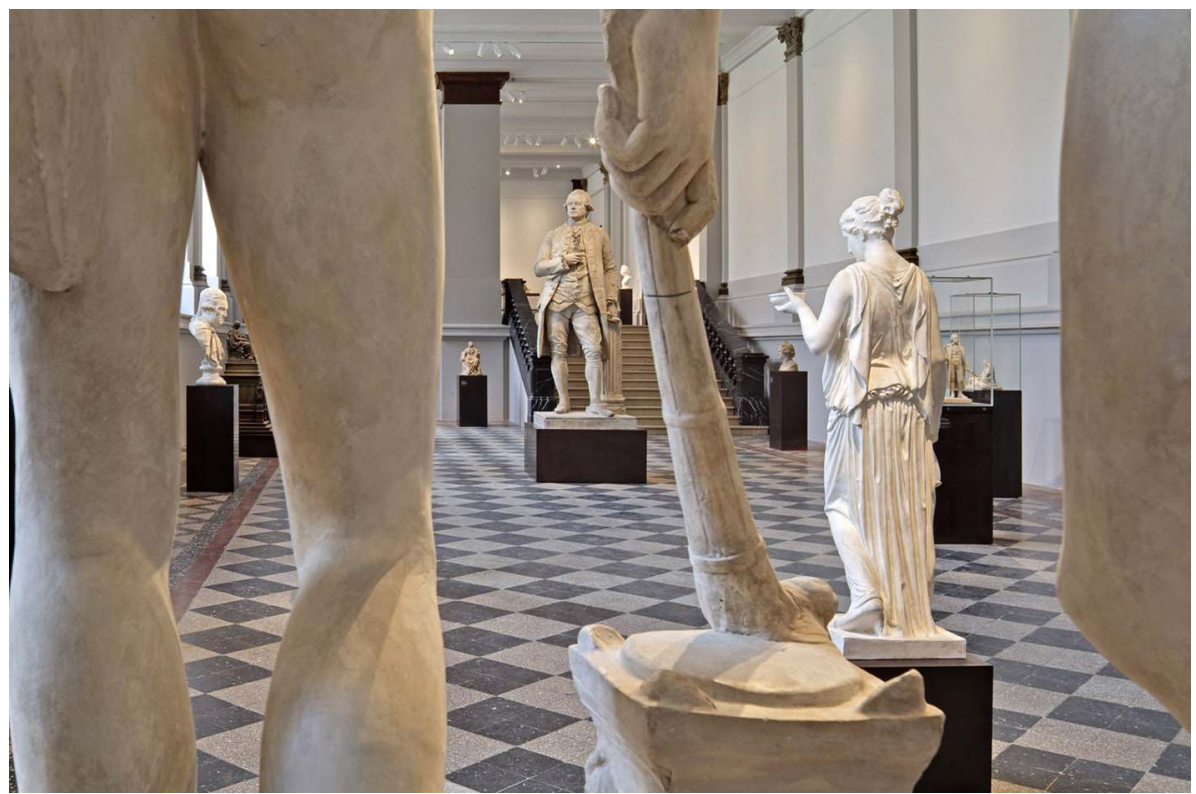

\section{La salle Klinger, un espace pour l'art de fin de siècle}

Par sa coloration forte et voluptueuse, la salle Klinger offre un contraste avec la salle de la mosaïque, même si le déroulé chronologique s’y poursuit (fig. 4). C'est avec une coloration semblable à celle de l'actuelle salle Klinger qu'il faut s'imaginer l'arrangement de la plupart des pièces du nouveau bâtiment d'exposition en 1889. Lors de la restauration de 2006, on s'est orienté vers l'état précédent encore conservé32.

La salle Klinger a reçu son nom en référence à l'œuvre le Drame du graphiste, peintre et sculpteur Max Klinger (1857-1920), œuvre qui y fut exposée à partir de $1904^{33}$. Aujourd'hui, celle-ci est présentée dans le hall des sculptures. On a opté pour une exposition commune de pièces des fonds de la Galerie des Nouveaux Maîtres et de la Collection des Sculptures, même s'il fallut faire des compromis sur l'accrochage des peintures. D'autres sculptures des années 1900 sont exposées dans cette pièce avec des œuvres de Max Klinger qui relèvent du symbolisme et de l'Art nouveau. Les travaux exposés se caractérisent par leur intérêt pour le corps humain, la mythologie et l'Antiquité, le féerique et le démoniaque. On y trouve des œuvres de Franz von Stuck (1863-1928), Hermann Prell (18541922), Sascha Schneider (1870-1927) et Artur Volkmann (1851-1941). Une grande partie des sculptures de la salle Klinger a été achetée par Georg Treu. Son envie de donner un nouvel élan à la sculpture colorée se reflète dans la peinture et la diversité des matériaux des pièces exposées, éléments qui représentent une

30. Astrid Nielsen et Saskia Wetzig, «Der Abguss der Gruppe von San Ildefonso und der Entwurf zum Goethe-Schiller-Denkmal Ernst Rietschels - zwei "klassische" Bildwerke im Mosaiksaal », Dresdener Kunstblätter, 54, 4, 2010 (= Themenheft : Das neue Albertinum), pp. 233-238, ici p. 233.

31. Concernant Ernst Rietschel : cat d'exp. Ernst Rietschel 1804-1861. Zum 200. Geburtstag des Bildhauers, sous la direction de Bärbel Stephan, Dresden, Skulpturensammlung der Staatlichen Kunstsammlungen, 23.10.2004-2.2.2005, München, Deutscher Kunstverlag, 2004

32. https://www.sib.sachsen.de/fileadmin/user_upload/pdf-dokumente/ broschueren/2012/2011_NL_Dresden_I_Albertinum.pdf (pp. 23-25) (consulté en 2016)

33. Reproduction dans : cat. d'exp., August Hudler in Dresden. Ein Bildhauer auf dem Weg zur Moderne, sous la direction d'Astrid Nielsen et Andreas Dehmer, Dresden, Albertinum, 19.9.201531.1.2016, Dresden, Sandstein-Verlag, 2015, p. 24. 
des fortes particularités de la Collection des Sculptures de Dresde ${ }^{34}$. Il s'agit de l'époque des œuvres totales par lesquelles les artistes se livraient au jeu des genres de manières très diverses. L'exposition de la salle Klinger donne l'impression que le Dresde de 1900 était le lieu privilégié de création, d'achat et d'exposition de la peinture et de la sculpture modernes ${ }^{35}$.

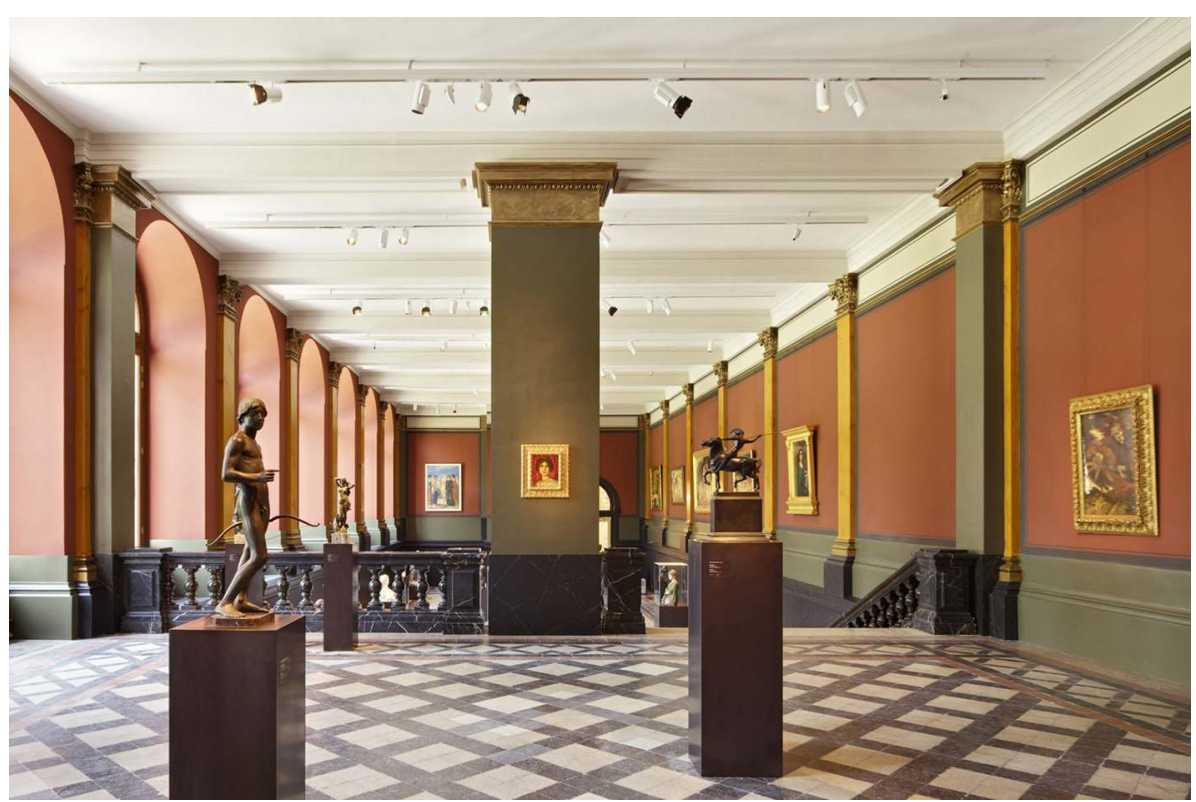

\section{Le hall des sculptures: de la sculpture de Rodin à aujourd'hui}

Le cheminement chronologique de l'exposition qui commence au premier étage dans les salles de la mosaïque et Klinger avec des œuvres de l'époque classique jusqu'au changement de siècle, se poursuit dans le hall des sculptures (fig. 5). Entouré de la Ruine de Thomas Scheibitz (né en 1968) et de la sculpture de Stephan von Huene (1932-2000) Sirenen Low / Der Gesang der Sirenen qui intègre musique et vidéo, un large spectre de la sculpture nationale et internationale, de Rodin à nos jours, se déploie dans le hall des sculptures. Au début de l'exposition chronologique se trouvent des œuvres d'Auguste Rodin, comme Le Penseur (plâtre, modèle agrandi, 1903), L'Homme au nez cassé (bronze, 1863-1864) ou le Buste hérö̈que de Victor Hugo (plâtre, 1896). Toutes ces acquisitions ont été faites du vivant de Rodin par Georg Treu ${ }^{36}$. Cette collection significative d'œuvres modernes classiques et en particulier d'œuvres de Rodin, Aristide Maillol (18611944), Constantin Meunier (1831-1905) et Wilhelm Lehmbruck est due en grande partie à Georg Treu. Après la mort de ce dernier et jusqu'à l'arrivée au pouvoir des national-socialistes en 1933, les directeurs successifs ont entretenu cette tradition d'achat d'importantes pièces contemporaines autant que les moyens et les circonstances le permettaient. Les pièces de l'après-guerre et jusqu'en 1989 reflètent l'histoire de l'Allemagne divisée. Les artistes de la RDA qui suivirent leur propre chemin artistique en marge de la doctrine d'État dominante sont fortement représentés, comme le montre l'exposition des pièces de Herman Glöckner (18891987), Wieland Förster (né en 1931) et Peter Makolies (né en 1936). Certaines de ces œuvres sont toutefois entrées dans la collection seulement après la révolution de 1989. Dans le même temps, la collection d'après-guerre s'agrandit des travaux d'artistes de l'Ouest comme Anthony Caro (1924-2013), Fritz Wotruba (1907-

34. Sur ce sujet entre autres : K. Knoll, « Treus Versuche zur antiken Polychromie und Ankäufe farbiger Plastik », Das Albertinum vor 100 Jahren, op. cit. note 10, pp. 164-168, cat. 151-168, 182 ; A. Nielsen, « Tradition und Innovation. Zur Skulptur in Dresden um 1900 », August Hudler in Dresden, op. cit. note 33, pp. 12-33, ici en particulier pp. 22-26.

35. Andreas Dehmer, «Im Bannkreis des ,Odolkönig' - Karl August Lingner, Franz von Stuck und der Erfolg symbolistischer Kunst in Dresden um $1900 \gg$, Dresdener Kunstblätter, 52, 4, 2008, pp. 244-257 ; A. Dehmer, Malerei des Fin de Siècle in der Dresdener Galerie, Dresden, SandsteinVerlag, 2010.

36. A. Nielsen et M. Woelk, « Rodin in Dresden. Erbe der Hellenen ? », Vor 100 Jahren. Rodin in Deutschland, op. cit. note 11, pp. 42-51, 179. 
1975) et Emil Cimiotti (né en 1927) mais s'enrichit aussi de prêts d'œuvres d'Henry Moore (1898-1986), Barbara Hepworth (1903-1975) et Per Kirkeby (né en 1938). Partant de la tradition de la sculpture figurative, l'éventail des œuvres englobe aujourd'hui des pièces, installations et travaux vidéos non figuratifs. À cela s'ajoute qu'à l'occasion de la réouverture de 2010 les fonds de la Collection des Sculptures ont été complétés de plusieurs œuvres contemporaines, parfois créées spécialement pour l'Albertinum, comme par exemple Ohne Titel (Ägypter) d’Ulrich Rückriems (né en 1938) placé à l'entrée.

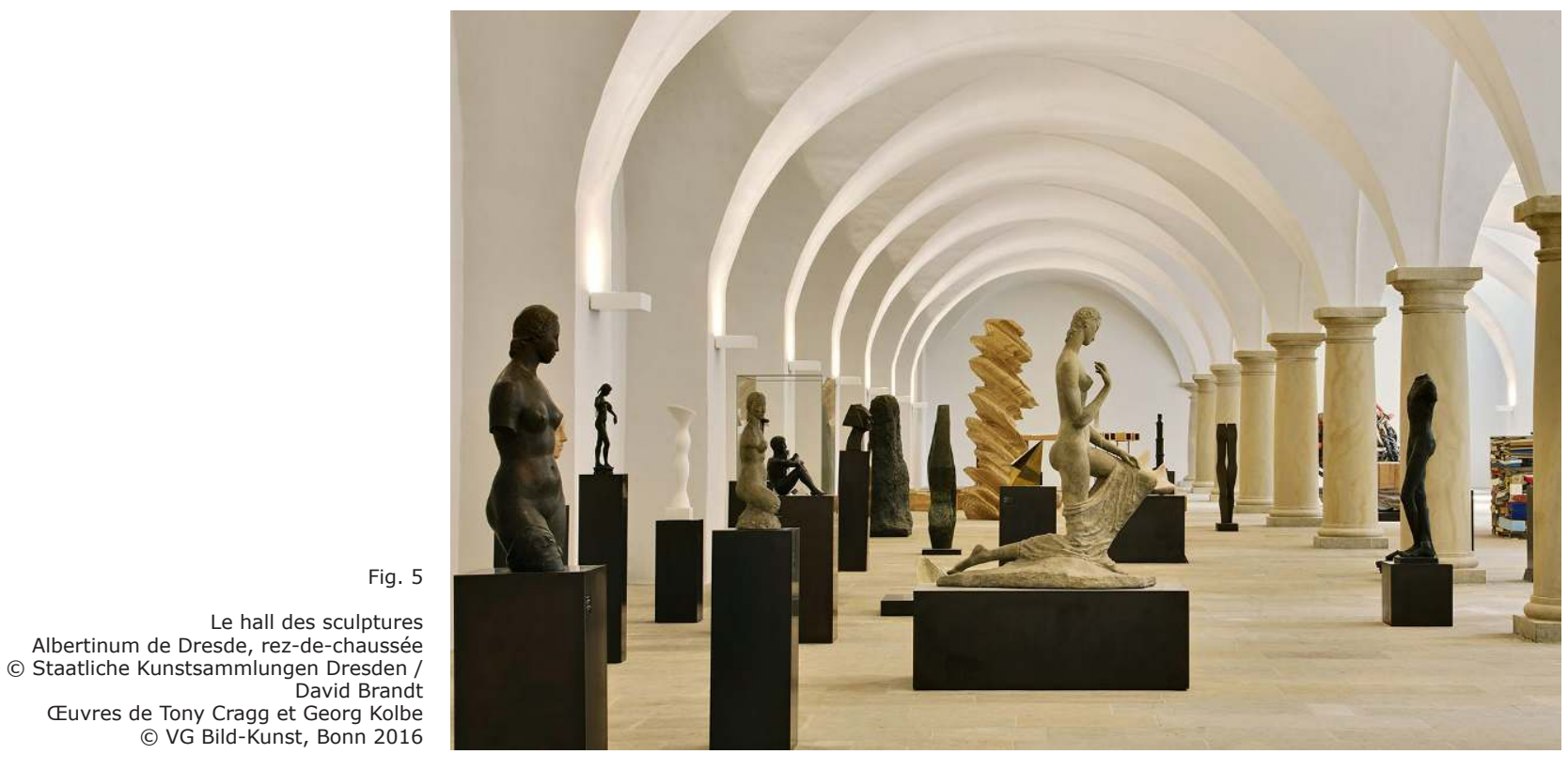

\section{Les socles}

Avec chaque nouvelle présentation se pose la question des socles et de leur matériau, tout au moins c'est le cas à Dresde. Durant les années précédant la Réunification, les œuvres de la collection d'antiques ont été placées sur des socles en grès et présentées dans de grandes vitrines à cloche de verre. Les pièces de la collection moderne étaient, quant à elles, posées sur des socles en bois peint. Après un long processus de recherche durant lequel il fut discuté de matériaux comme le bois, la pierre ou le ciment, il a été décidé que les nouveaux socles seraient en métal, plus exactement en bronze brun, et contiendraient un encastrement pour les cartels. Plusieurs tailles de socle ont été élaborées pour les collections permanentes et pour de futures expositions. Les vitrines pour les œuvres fragiles, ou à protéger, ont été fabriquées sur mesure et les cloches de verre sont posées sur la bordure supérieure du socle en bronze de sorte qu'elles se ferment à la même hauteur. Les plus lourds pèsent entre 100 et $279 \mathrm{~kg}$ et leur construction garantit une statique particulièrement sûre, l'inconvénient, en revanche, tient dans la difficulté à déplacer de tels objets ${ }^{37}$.

\section{De l'utilisation de la cour intérieure et des plans pour l'avenir de l'Albertinum}

La cour lumineuse et généreuse invite à être utilisée comme un espace d'exposition, mais comme elle est également louée comme un lieu de manifestations culturelles pour des raisons financières, elle ne se prête pas vraiment à une présentation permanente (fig. 6). Dernièrement, on y a exposé les Berserker de la berlinoise Stella Hamberg (née en 1972) ou le travail d'une autre artiste berlinoise Ursula Sax (née en 1935) qui a su utiliser l'espace aérien de la salle. L'installation dont l'exposition s'est terminée en septembre 2015 fut créée

37. Essais sur la stabilité des socles en collaboration avec I'Université Technique de Dresde : https://baublog.file1.wcms.tu-dresden.de/2010/07/06/rempeln-fur-mehr-sicherheit-im-albertinum/ (consulté en janvier 2016). 
par Fernando Sánchez Castillo (né en 1970) et s'intitulait Made in China. Ce fut d'ailleurs l'une des premières muséographies de Hilke Wagner, qui est entrée dans ses fonctions de directrice de l'Albertinum de Dresde en novembre 2014. Cette dernière envisage à l'avenir de renforcer le mélange peinture-sculpture dans l'exposition permanente et élargit pour ce faire le concept de la sculpture à la sculpture cinétique, à la sculpture sonore et à l'art vidéo. Les prémices de cette évolution pourront être vues avec l'exposition Rosa Barba. Spaces for species (and pieces) ${ }^{38}$. Rosa Barba commença très tôt à travailler la sculpture en film et ne se limita pas à représenter la sculpture comme un objet. Le propos central de son travail conceptuel prend la forme de projections d'images avec un projecteur qui devient lui-même l'objet de la mise en scène artistique.

Fig. 6

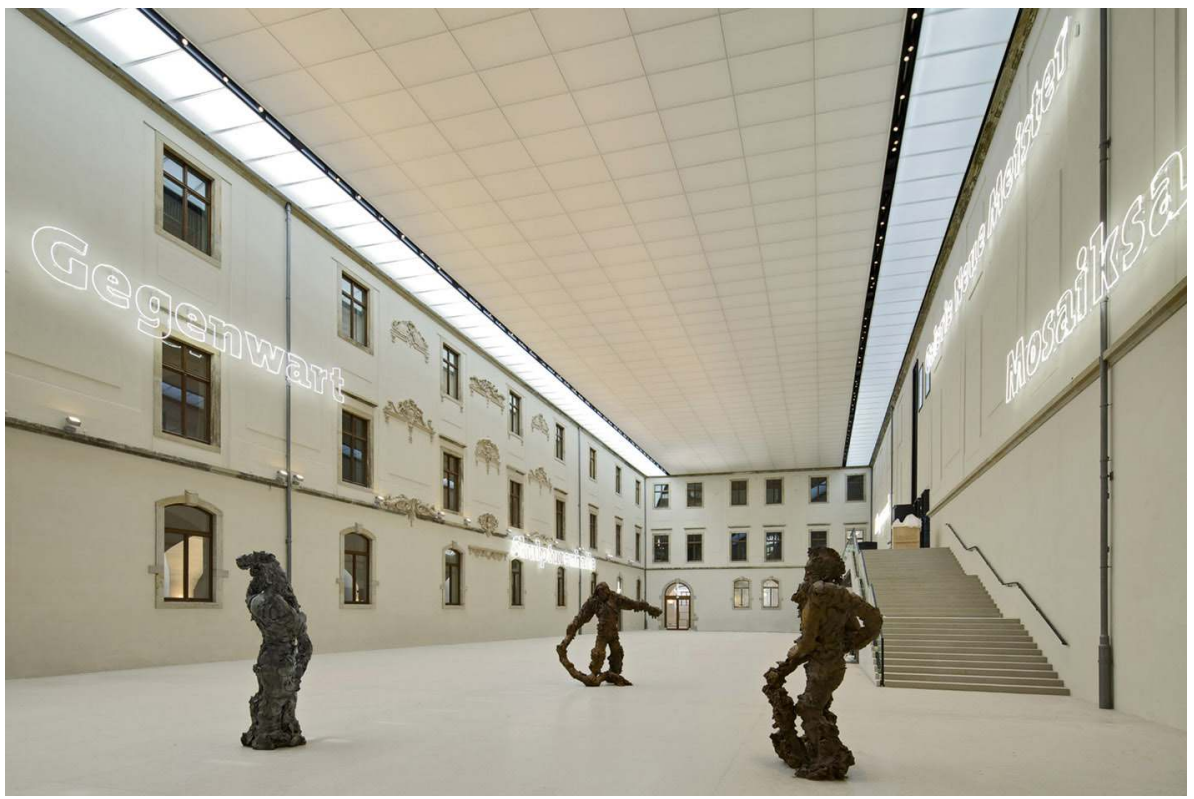

\section{L'avenir de la collection d'antiques}

Le nouvel agencement de l'Albertinum représentait le plus grand changement pour la Collection des Sculptures, puisque cela signifiait dès le début une conception globale tout à fait neuve. Les antiques, pour lesquels Dresde est connue, ne sont montrées depuis 2010 que dans le cadre de l'exposition du dépôt. Des sculptures choisies sont mises en scène pour les visiteurs, comme dans le " dépôt de verre " au niveau de l'entrée de plain-pied depuis la place Georg Treu, dans l'espace qui héberge les sculptures de grand format. Le second dépôt exposé, au premier étage, montre les sculptures de l'Antiquité jusqu’à l'époque baroque, ainsi que quelques pièces choisies du baroque à nos jours. Alors que les salles d'exposition de l'Albertinum devraient se concentrer sur la nouvelle partie de la collection, c'est-à-dire des Lumières à l'époque contemporaine, l'ancienne partie, de l'Antiquité à l'époque baroque, devrait être exposée au rez-de-chaussée du hall est du bâtiment des galeries de Gottfried Semper dans le Zwinger de Dresde. Ce hall avait d'ailleurs été construit pour les sculptures, et particulièrement pour la collection de moulages ${ }^{39}$. L'inauguration de cette exposition devrait avoir lieu en 2019. Le nouvel agencement accordera la beauté des œuvres d'art avec l'architecture de Semper. L'art antique devrait être transmis au visiteur d'aujourd'hui de manière à ce que sa teneur soit bien comprise et que son esthétique en devienne convaincante ${ }^{40}$. 38. http://www.skd.museum/de/sonderausstellungen/archiv/rosa-barba/index.html (consulté
en février 2016).

39. Sur ce sujet entre autres : M. Kiderlen, «Einführung 》, Die Sammlung der Gipsabgüsse, op. cit. note 8, pp. 15-32, ici pp. 23 seq. avec repr.

40. Sur ce sujet : K. Knoll, « Skulpturensammlung », Jahrbuch der Staatlichen Kunstsammlungen Dresden 2014 (à paraître). 
Les questions relatives à l'exposition et la présentation de la sculpture demeurent donc à Dresde un sujet d'actualité et ce, pour quelques années encore. Les différents fonds du musée seront présentés en plusieurs endroits ; il n'en demeure pas moins que la Collection des Sculptures reste une collection dont la croissance fut rythmée par les vicissitudes de l'histoire.

Traduit de l'allemand par Coralie Zermatten

\section{L'auteur}

Formée en histoire de l'art, en langues romanes et en lettres modernes allemandes à l'Université Christian Albrecht de Kiel, Astrid Nielsen a également effectué plusieurs séjours à l'étranger. Depuis 1999, elle travaille pour les Collections d'État de Dresde et depuis 2001 à la Collection des Sculptures. En 2006, elle était commissaire de l'exposition Vor 100 Jahren. Rodin in Deutschland avec Moritz Woelk en collaboration avec le musée Rodin et le Bucerius Kunst Forum de Hambourg, en 2009 pour l'exposition Wieland Förster zum 80. Geburstag. Alle Werke seiner Stiftung et enfin en 2015 pour August Hudler in Dresden. Ein Bildhauer auf dem Weg in Moderne. Par ailleurs, elle a pris part au Museum Experts Exchange Program (MEEP) en Chine et a effectué un séjour scientifique au Victoria \& Albert Museum de Londres. Astrid Nielsen a tenu de nombreuses communications et rédigé plusieurs contributions sur la sculpture des $\mathrm{XIX}^{\mathrm{e}}$ et $\mathrm{XX}^{\mathrm{e}}$ siècles, mais aussi sur la sculpture en RDA.

\section{The author}

Having studied art history, Romance languages and modern German literature at Christian-Albrechts-Universität zu Kiel, Astrid Nielsen also made many trips abroad. Since 1999, she has been working for the Staatliche Kunstsammlungen Dresden and for the Skulpturensammlung since 2001. In 2006 she curated the exhibition Vor 100 Jahren. Rodin in Deutschland with Moritz Woelk in collaboration with the Musée Rodin and the Bucerius Kunst Forum in Hamburg, in 2009 she curated the exhibition Wieland Förster zum 80. Geburtstag. Alle Werke seiner Stiftung and in 2015 she curated the exhibition August Hudler in Dresden. Ein Bildhauer auf dem Weg in Moderne. She also took part in the Museum Experts Exchange Program (MEEP) in China and a effectué a scholarly residence at the Victoria \& Albert Museum in London. Astrid Nielsen has delivered many lectures and written several article on nineteenth-and twentieth-century sculpture, as well as on sculpture in the GDR. 